\title{
PLANETARY CAMERA OBSERVATIONS OF THE DOUBLE NUCLEUS OF M31 ${ }^{1}$
}

\section{TOD R. LAUER}

Kitt Peak National Observatory, National Optical Astronomy Observatories, ${ }^{2}$ P. O. Box 26732, Tucson, Arizona 85726 Electronic mail: lauer@ noao.edu

\section{S. M. FABER}

UCO/Lick Observatories, Board of Studies in Astronomy and Astrophysics, University of California, Santa Cruz, Santa Cruz, California 95064

Electronic mail: faber@ helios.ucsc.edu

\section{EDWARD J. GROTH}

Physics Department, Jadwin Hall, Princeton University, Princeton, New Jersey 08544 Electronic mail: groth@pupgg.princeton.edu

\section{EDWARD J. SHAYA}

Department of Physics and Astronomy, University of Maryland, College Park, Maryland 20742

Electronic mail: shaya@umaip.umd.edu

\section{Bel CAMPBell}

Department of Physics and Astronomy, University of New Mexico, 800 Yale Boulevard, N. E., Albuquerque, New Mexico 87131

Electronic mail: bel@unmb.unm.edu

\section{ARTHUR CODE}

Wasburn Observatory, University of Wisconsin, 475 N. Charter Street, Madison, Wisconsin 53706 Electronic mail: code@uwfpc.span

\section{DOUglas G. CURRIE}

Department of Physics and Astronomy, University of Maryland, College Park, Maryland 20742 Electronic mail: currie@img.umd.edu

\section{WILLIAM A. BAUM}

Astronomy Department, University of Washington, FM-20, Seattle, Washington 98195 Electronic mail: baum@astro.washington.edu

\section{S. P. EWALD}

Space Telescope Science Institute, 3700 San Martin Drive, Baltimore, Maryland 21218 Electronic mail: ewald@stsci.edu

\section{J. JEFF HESTER}

Department of Physics and Astronomy, Arizona State University, Tempe, Arizona 85287 Electronic mail: jih @ cosmos.la.asu.edu

\section{JON A. HOLTZMAN}

Lowell Observatory, 1400 Mars Hill Road, Flagstaff, Arizona 86001 Electronic mail: holtz@ lowell.edu

\section{JEROME KRISTIAN}

The Observatories, Carnegie Institution of Washington, 813 Santa Barbara Street, Pasadena, California 91101 Electronic mail: jak@soll.gps.caltech.edu

\section{ROBERT M. LIGHT}

Infrared Processing and Analysis Center, 100-22, California Institute of Technology, Pasadena, California 91125 Electronic mail: light@ipac.caltech.edu

\section{ROger LyNDS AND EARL J. O'NeIL, JR.}

Kitt Peak National Observatory, National Optical Astronomy Observatories, P. O. Box 26732, Tucson, Arizona 85726 Electronic mail: lynds@ noao.edu, oneil@ noao.edu 
JAMES A. WESTPHAL

Division of Geological and Planetary Sciences, 170-25, California Institute of Technology, Pasadena, California 91125 Electronic mail: jaw @ sol1.gps.caltech.edu

Received 1993 April 26; revised 1993 June 7

\begin{abstract}
$V$ - and I-band HST Planetary Camera images of the great spiral galaxy in Andromeda, M31, show that its inner nucleus consists of two components separated by 0.49 . The outer isophotes of the nucleus at $1{ }^{\prime \prime} 4<r<3$." 0 are elongated, but are concentric with the M31 bulge. The nuclear component with the lower surface brightness, $\mathbf{P} 2$, is also coincident with the bulge photocenter to $\sim 0$ ".05; we argue that it is at the kinematic center of the galaxy. The brighter nuclear component, P1, is well resolved and corresponds to the nuclear core imaged by Stratoscope II. The central $V$-band luminosity density for P1 is $5 \times 10^{4} L_{\odot} \mathrm{pc}^{-3}$. P2 is highly elongated and has a shallow cusp. Its central $V$-band luminosity density is $>2 \times 10^{4} L_{\odot} \mathrm{pc}^{-3}$. The total nucleus cannot be modeled as the superposition of two systems each with elliptical and concentric isophotes. The dark zone between the two peaks is deep and is either evidence for strong dust absorption or that the nuclear components are steeply truncated in this region. No strong changes in the $V-I$ color are observed over the nucleus, however. Thus if dust absorption generates the asymmetric nuclear morphology, the dust grain size must be unusually large or the dust optical depth must be extremely high. Alternatively the higher surface brightness, off-center nuclear component P1 may be a separate stellar system. The nuclear velocity dispersion profiles of Dressler \& Richstone [ApJ, 324, 701 (1988)] and Kormendy [ApJ, 325, 128 (1988)] already show that P1 cannot be a dynamically cold system such as a globular cluster. Requiring P1 to survive destruction by a central black hole in M31 may also lead to lower limits on its mass-to-light ratio. This hypothesis requires us to be looking at M31 at a special time; however, the lifetime of P1 against orbital decay may be substantially longer than naive dynamical friction arguments would indicate. Under this picture, we argue that $\mathbf{P} 1$ is more likely to be a cannibalized galactic nucleus, possibly having its own central black hole.
\end{abstract}

\section{INTRODUCTION}

At the prime focus of a large telescope, the M31 nucleus looks like a star surrounded by the smooth nebulousity of the underlying bulge. It is the prototype of the central luminous star clusters found in many galaxies-its appearance lending the name "stellar nuclei" by which such objects are known (see Lauer 1989a and Kormendy \& Djorgovski 1989, for reviews). It has long been known (Redman \& Shirley 1937) that the M31 nucleus is highly compact, making it difficult to probe its structure from the ground. The Hubble Space Telescope (HST) now provides the resolution required to explore the structure of nearby nuclear star clusters, a problem that is especially interesting for M31 as its nucleus may harbor a black hole of $1-5 \times 10^{7} \mathscr{M}_{\odot}$ (Dressler 1984; Dressler \& Richstone 1988; Kormendy 1988; Richstone et al. 1990).

The first good look at the structure of the M31 nucleus was provided by 0 ". 2 resolution photographs obtained by Stratoscope II (Light et al. 1974). The Stratoscope II images clearly resolved the nucleus, showing it to have a core radius (location at which the surface brightness falls to

${ }^{1}$ Based on observations with the NASA/ESA Hubble Space Telescope, obtained at the Space Telescope Science Institute, which is operated by AURA, Inc., under NASA Contract No. NAS 5-26555.

${ }^{2}$ Operated by the Association of Universities for Research in Astronomy, Inc., under cooperative agreement with the National Science Foundation. half of its central value) of 0.48 , or $1.8 \mathrm{pc}$, adopting the distance to M31 of $770 \mathrm{kpc}$ (Freedman \& Madore 1990). The same images showed the nucleus to be elongated and asymmetrical as well, having a low intensity extension on one side of the bright peak. Light et al. (1974) raised the possibility that they were looking at the effects of dust obscuration cutting into a more extended nucleus, but unfortunately their exposures were too shallow to examine the outer structure of the nucleus, let alone the surrounding M31 bulge. Nieto et al. (1986) imaged the nucleus at 0 "6 resolution in the near-UV, confirming the broad appearance of the nucleus evident in the Stratoscope II images, but also found the nucleus to be offset from the bulge by 0"4. Johnson (1961), Kent (1983), as well as Nieto et al. (1986) have all argued for the presence of dust close to the nucleus; however, the offset of the nucleus with respect to the bulge is still present in $K$-band images (Mould et al. 1989), arguing against the asymmetry as being due to absorption by a foreground patch of dust, or perhaps any form of dust with standard properties.

Despite the apparent displacement of the nucleus from the bulge, both Dressler \& Richstone (1988), and Kormendy (1988) find that the rapid central rise in stellar velocity dispersion and rotation, which they take as evidence for a massive black hole, peaks at the bulge center rather than the nucleus brightness peak. Indeed, the kinematic profiles superficially appear symmetric over the same region that the starlight appears asymmetric. Dressler \& 
Richstone (1988), and Richstone et al. (1990) assume that the nucleus is an ellipsoidal star cluster and conclude $\mathscr{M}_{\bullet} \approx 4-5 \times 10^{7} \mathscr{M}_{\odot}$. Kormendy (1988) gets similar black hole masses under the same dynamical assumptions, but also finds $\mathscr{M}_{\bullet} \approx 1 \times 10^{7} \mathscr{M}_{\odot}$ if the nucleus instead is actually a rapidly rotating disk seen nearly edge on. Finally, Gerhard (1988) raises the possibility that the nucleus is actually a bar seen end on, and that the apparent rapid rotation may really be due to stellar streaming with no need for a black hole at all.

We obtained high signal-to-noise $V$ - and $I$-band images of the M31 nucleus with the HST Planetary Camera to constrain the plausible range of nuclear morphological models. We note that M31 has been imaged previously by HST (King et al. 1992), but in the far-UV and with very low signal to noise. From our new images, we find that the nucleus is asymmetric, being split into two components, rather than simply being a single cluster offset from the bulge. We investigate two hypotheses for the origin of the asymmetry, which in part follow similar ideas presented by Dressler \& Richstone (1988). In the first case we consider the effects of central dust obscuration on the nucleus. In the second case we consider the possibility that brighter nuclear component is actually a separate stellar system. We find either explanation somewhat problematic, but their consideration motivates the key goal of our analysis, which is to improve the objective description of this complex system.

\section{OBSERVATIONS AND ANALYSIS}

\subsection{Observations and Basic Reduction}

HST observations of M31 were obtained on day 187 of 1991 with the PC or high resolution mode of the Wide Field/Planetary Camera (WFPC). Briefly, the PC comprises four CCD cameras imaging a contiguous $66^{\prime \prime} \times 66^{\prime \prime}$ field of view. Each CCD has a $800 \times 800$ pixel format with 0 "044 per pixel. Further description of the camera is presented by Griffiths (1989). One $100 \mathrm{~s}$ and four $300 \mathrm{~s}$ exposures were obtained with filter F555W, which corresponds roughly to the Johnson $V$ band, and two $400 \mathrm{~s}$ exposures were obtained with filter F785LP, which corresponds roughly to the Johnson $I$ band. The telescope was guided in coarse track during the exposure sequence. The nucleus was positioned in the center of CCD PC6; for the present analysis only the data from PC6 were used. The position angle of the PC6 CCD column was $70^{\circ}$.

At the time of the observations, calibration of the WFPC was still in a preliminary state; however, all reduction steps outlined by Lauer (1989b) could be completed. The flat fields for F555W and F785LP were generated from several "streak" frames and gave generally excellent results. A number of compact defects remained in the images due to material on the CCD field flatteners, but fortunately none of them affected the nucleus itself. A blocked column fell two pixels away from the brightest portion of the nucleus in the F555W exposures, but the intensity gradient at that location was very shallow, and the column could be easily interpolated across. Both F785LP images

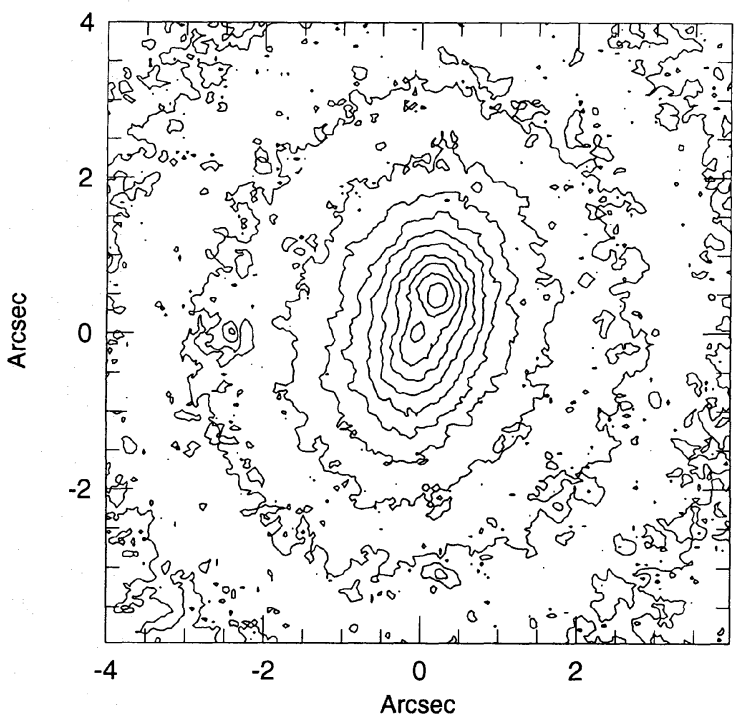

FIG. 2. Contour map of the deconvolved $V$ image. Contour steps are shown every $0 \div 25$ step in surface brightness; the brightest contour shown is at $\mu_{V}=13.50 \mathrm{mag} / \mathrm{sq}$. arcsec. The map has normal celestial handedness. The vertical axis has been rotated $70^{\circ}$ counterclockwise from north.

had a saturated pixel at the peak of the nucleus; again the gradient in the affected region was very shallow, and it was easy to estimate the brightness at the pixel location. Cosmic rays were rejected by comparison of the images, and a composite image was produced for each filter by adding the individual exposures. The nuclear centroids of the individual images agreed at the milliarcsecond level, and the images could be added directly without any shifting. The peak nuclear signal was $7.2 \times 10^{4} e^{-} /$pixel in the final F555W image, and 5.0 $\times 10^{4} e^{-} /$pixel in the F785LP image. In the discussion that follows, we have assumed zeropoints of $23^{\mathrm{m}} \cdot 10$ and $21^{\mathrm{m}} \cdot 45$ corresponding to $1 \mathrm{DN} / \mathrm{s}$ in the F555W and F785LP filters, respectively, where $1 \mathrm{DN}$ is 7.6 $e^{-}$, and is the basic WFPC A/D data number quantum.

An accurate Point Spread Function (PSF) is crucial to the image analysis, given the severe spherical aberration present in the HST primary (Burrows et al. 1991). We obtained a fast exposure of a bright star in $V$ and $I$ within 50 pixels of the CCD location of the nucleus shortly after the M31 exposures were complete. We further supplemented our knowledge of the PSF with other fast star exposures obtained within a few days of the M31 exposures. In general, knowledge of spacecraft jitter during the exposures is also required; however, the nucleus of M31 is sufficiently well resolved that we can ignore the minor effects of jitter in the analysis that follows.

\subsection{Morphology and Location of the Nucleus}

The central portions of the reduced $V$ and $I$ images after 80 iterations of Lucy-Richardson deconvolution (Lucy 1974; Richardson 1972) are presented in Fig. 1 (Plate 62). A contour map of the nucleus in $V$ is shown in Fig. 2. The present HST images clearly go beyond the Strato- 
scope $I I$ images in offering new information about the nucleus. We now see that the nucleus actually has two brightness peaks. The dark zone separating the peaks looks more structured than a simple saddle defined by the peaks, as it appears to have a somewhat straight and abrupt edge on the side of the brighter peak that runs nearly perpendicular to the long axis of the nucleus. At first glance the zone strongly resembles a dust lane, a point that we will consider in detail later. The peak with the higher surface brightness, which we designate P1, corresponds to the feature resolved by Stratoscope II and King et al. (1992). The second peak, P2, lies within the faint asymmetric extension from P1 noted by Light et al. (1974). The position angle of the line joining the peaks is $43^{\circ} \pm 1^{\circ}$, while the position angle of the nuclear isophotes for $0.5<r<1.0$ is about $63^{\circ}$, showing a clear twist of the innermost portion of the nucleus from its outer portions. The position angle of the P1-P2 axis is bracketed by that of the M31 disk, $38^{\circ}$ (de Vaucouleurs 1958), and that of the bulge, $48^{\circ} \pm 3^{\circ}$ (Kent $1983)$. Further, in the $V$ image at least, both peaks individually appear to be elongated along the P1-P2 axis at the highest surface brightness levels (see the upper left panel in Fig. 1). As a consequence of the twist of the P1-P2 axis from that of the outer nucleus, neither peak appears to be quite reflection symmetric about the major axis-each appears to slightly fan out on opposite sides of the major axis from each other. The steeper falloff on the left side (as in Figs. 1 and 2) of $\mathrm{P} 2$ in fact appears to be a continuation of the dark zone separating the two peaks.

We will consider color gradients in the nucleus later, but for now, it's interesting to contrast the $V$ and $I$ images in Fig. 1. P2 is clearly much sharper in $V$ than $I$, but oddly P1 looks more compact and less elongated in $I$. The resolution of the deconvolved images is close to the nominal diffraction limited performance of $H S T$ and is clearly sharper in $V$ than $I$. This may account for the different appearance of $\mathrm{P} 2$, but the behavior of $\mathrm{P} 1$ is more confusing. In passing, we note that there are no strong brightness gradients present in the nucleus, so the deconvolved images should produce quantitatively useful results (see Lauer et al. 1992).

Our first analysis issue is relating the positions of the nuclear components to the bulge centroid. From the ground, one might assume that the nucleus is a star cluster merely displaced from the bulge center. In contrast, the first impression that some of us had from the HST images was that the nucleus was an elongated structure with its true center (and that of the bulge) hidden behind a dust torus. It now appears that the true center of the bulge, and probably that of the nucleus itself, corresponds to the dimer peak, P2. The separation of P2 from P1 is 0"49 \pm 0 ". 01 or $1.8 \mathrm{pc}$, based on centroids measured in $3 \times 3$ pixel boxes centered on the brightest pixel in each peak in the $V$-band image. For comparison, Nieto et al. (1986) find a $\sim 0$. 4 nucleus-bulge centroid difference (with the bulge offset roughly in the P2 direction), as does Mould et al. (1989). Kormendy (1988) finds the kinematic center of rotation to be offset from the photocenter by $0.3 \pm 0$ " 1 , similar to Dressler \& Richstone (1988), who find a $\sim 0$ ".5

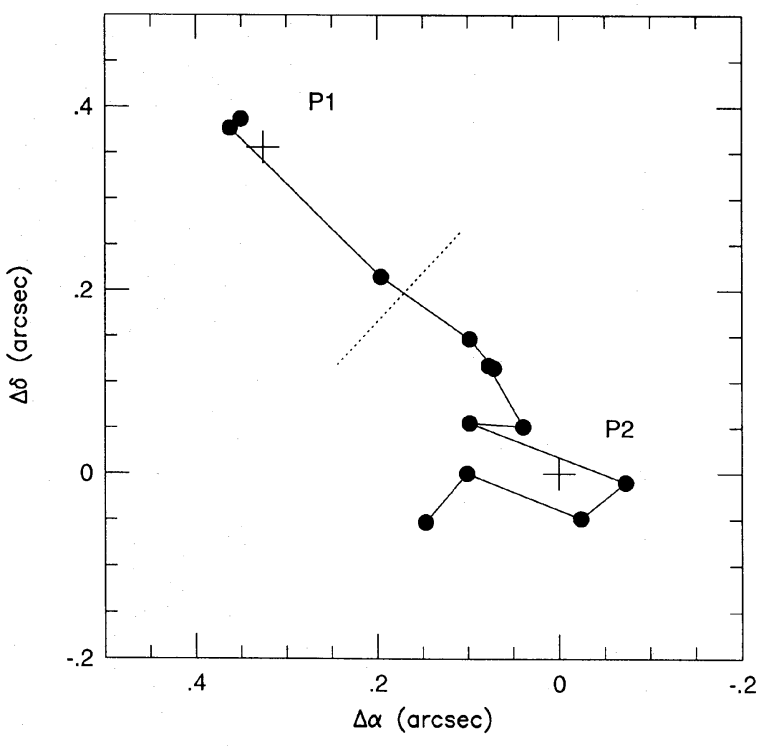

FIG. 3. Map of isophote centers relative to $P 2$ in celestial coordinates. The line connects steps of $0 \cdot 25 / \mathrm{sq}$. arcsec in isophote brightness. The first point at $\mathrm{Pl}$ corresponds to $\mu_{V}=13 \mathrm{~m} \cdot 25 / \mathrm{sq}$. arcsec. The last point corresponds to $\mu_{V}=16^{\mathrm{m}} \cdot 00 / \mathrm{sq}$. arcsec. The dotted line indicates the center of the dark zone.

offset, again in the P2 direction. These results imply that the massive black hole proposed to explain the M31 nuclear dynamics should be located near P2. For comparison, the brightness profile between $\mathrm{P} 1$ and $\mathrm{P} 2$ reaches its minimum 0"26 from P2.

Further analysis of our own images shows that P2 is close to the bulge center. In Fig. 3 we plot the isophote centers as a function of surface brightness in the deconvolved $V$ image. These reflect the center of mass of all pixels within $\pm 0^{\mathrm{m}} \cdot 125 / \mathrm{sq}$. arcsec of the specified surface brightness (extraneous pixels at large distances from the nuclear isophotes were excluded). Individual isophotes corresponding to $\mu_{V} \geqslant 14 \cdot 75 / \mathrm{sq}$. arcsec are clearly centered to within 0 ". 1 of P2. Indeed, it is noteworthy how quickly the asymmetry of the nucleus disappears as one moves out from the center; the isophote corresponding to $\mu_{V}=14^{\mathrm{m}} \cdot 75 / \mathrm{sq}$. arcsec has a semimajor axis of only 1.05 . We note that isophotes at this brightness level are still very much part of the nucleus. In Fig. 4 we plot the surface brightness profile centered on P2 (details on measurement of the profile are presented in Sec. 2.4). The inflection point corresponding to the rise of the nuclear component above the bulge occurs at $\mu_{V} \sim 15^{\mathrm{m}} \cdot 8 / \mathrm{sq}$. arcsec, about $3^{\prime \prime}$ out from P2. This effect can also be seen in Fig. 5, where we plot isophote ellipticity as a function of radius. One sees that the nuclear flattening of the isophotes also sets in at 3 ", well outside of the radius where the asymmetry associated with $\mathbf{P} 1$ begins. The fundamental stellar system that constitutes the nucleus is thus well centered on the bulgethe central asymmetry occurs interior to the nucleus itself.

As a simple check that $\mathrm{P} 2$ is the true center of M31, we took a five pixel wide cut along the major axis (position angle $43^{\circ}$ ) of the nucleus (shown in Fig. 6) and then sub- 


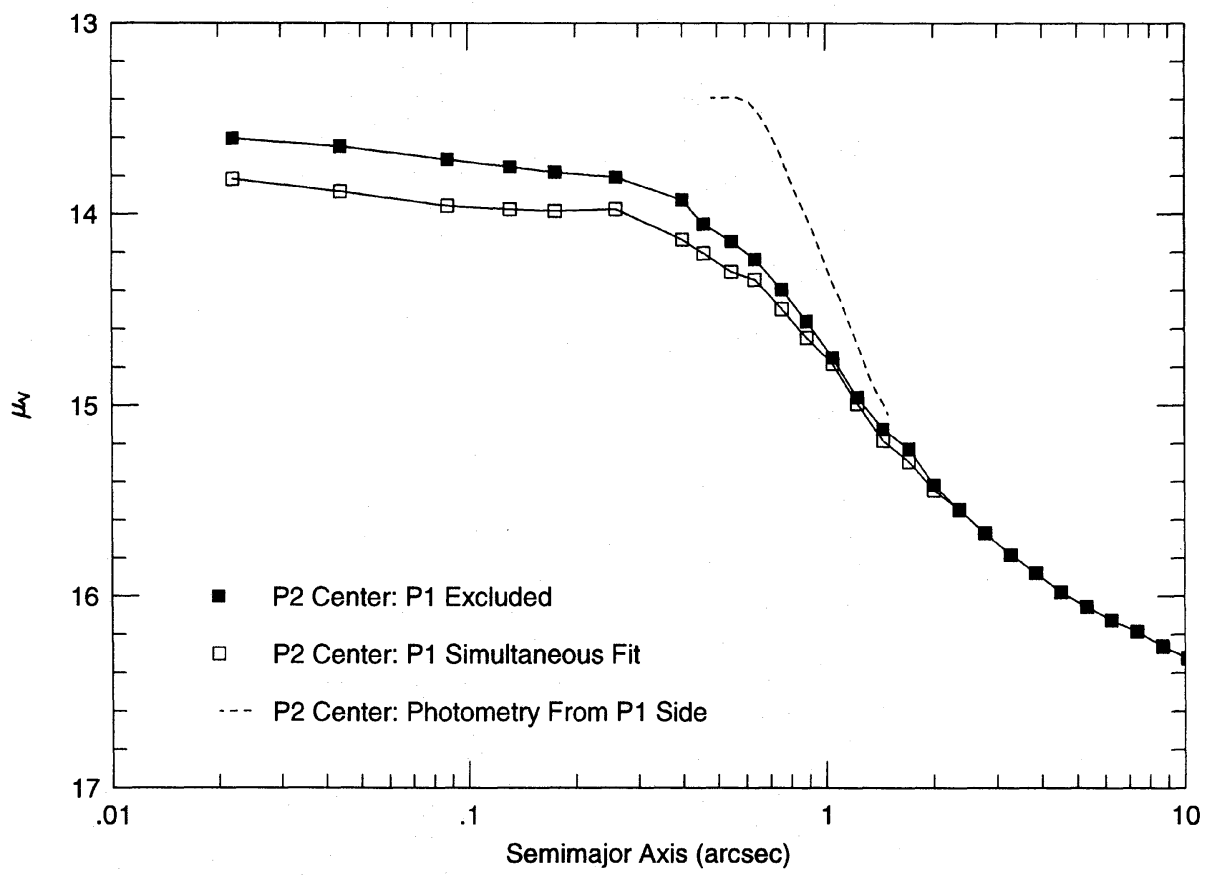

FIG. 4. Deconvolved $V$-band major-axis surface brightness profiles of the M31 nucleus with P2 selected as the center. No correction for galactic absorption has been applied. The solid symbols show the P2 plus bulge profile derived with the region associated with the excess brightness around P1 simply excluded. The dashed line shows the photometry derived from the P1 side instead. The lowest trace (open symbols) is the P2 plus bulge brightness profile derived with a simultaneous fit under the assumption that P1 and P2 are distinct but overlapping systems unaffected by dust. The corresponding P1 profile is shown in Fig. 9.

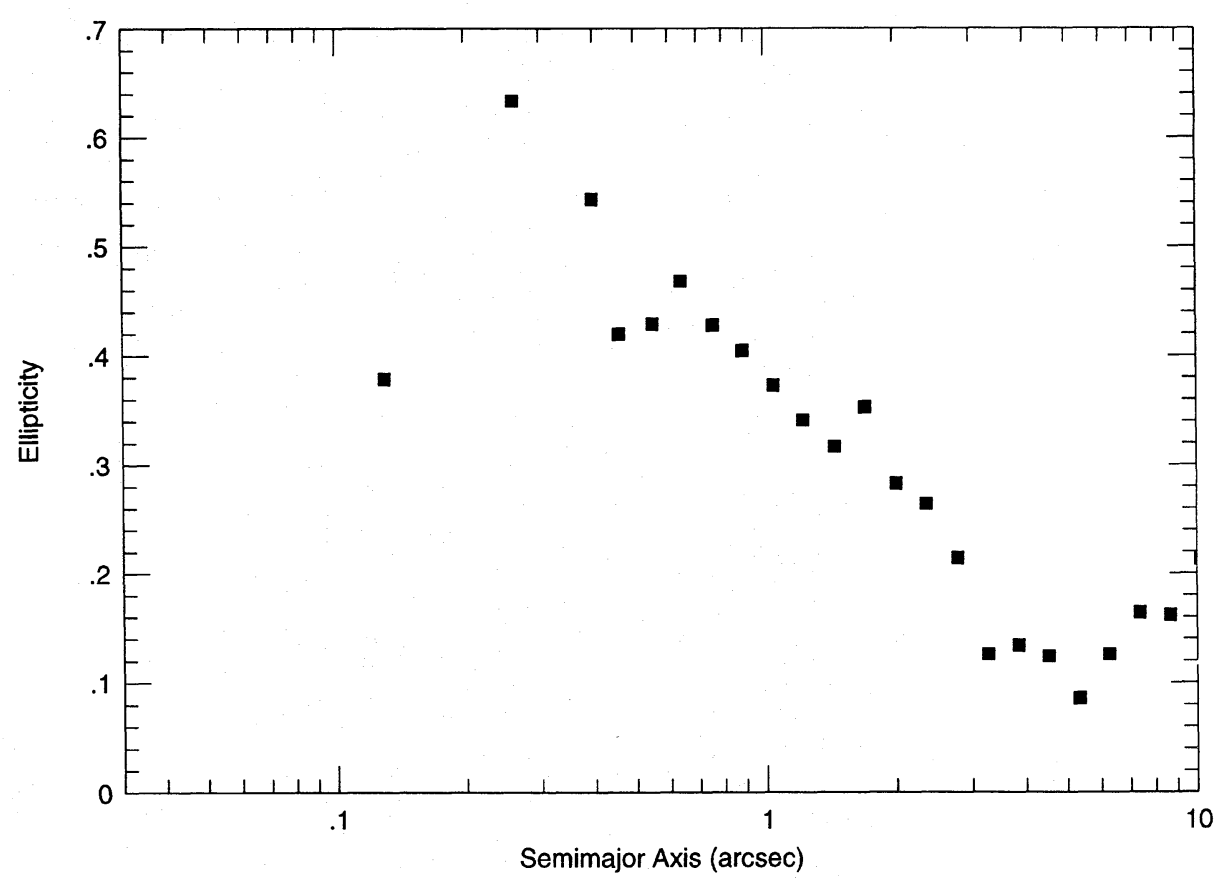

FIG. 5. $V$-band isophote ellipticity centered on P2. P1 was excluded from the fit. 


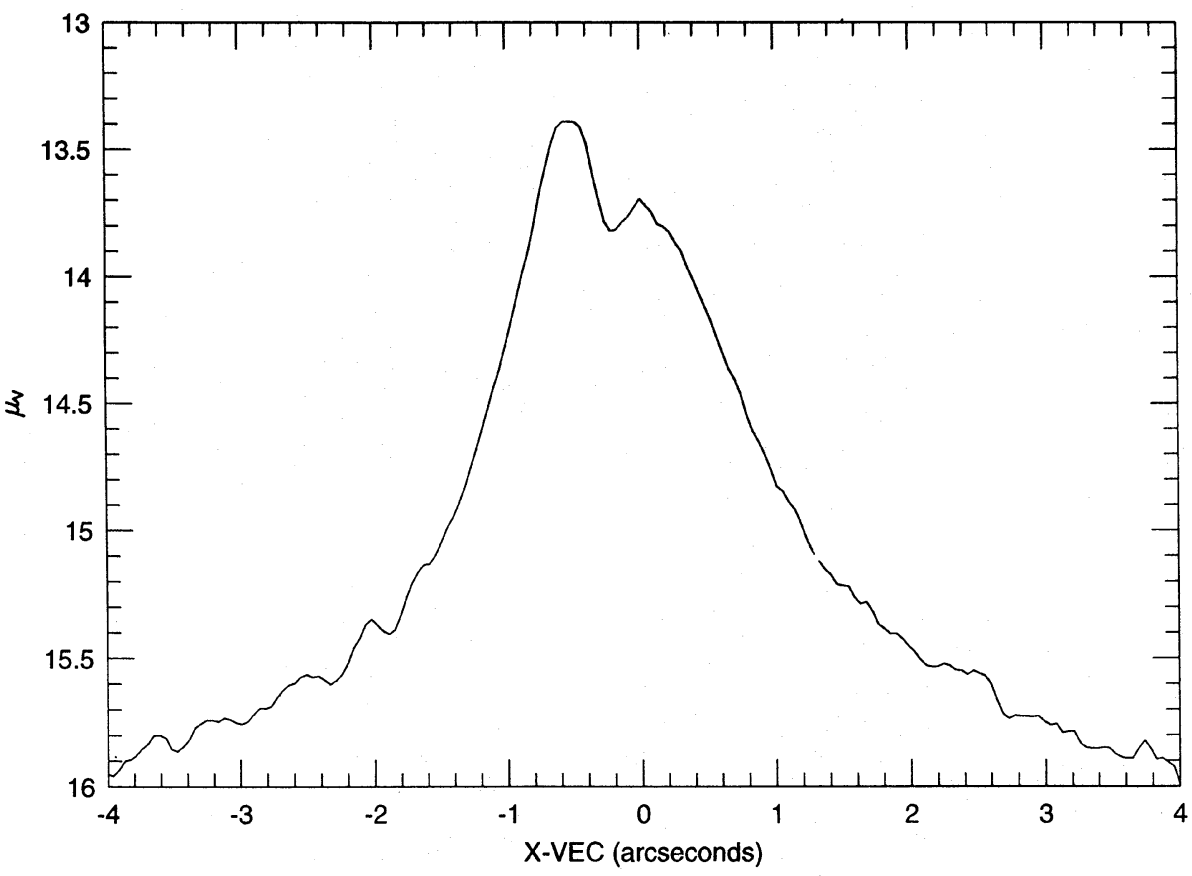

FIG. 6. The $V$-band surface brightness measured from a slice 5 pixels wide at PA $43^{\circ}$ containing $\mathrm{P} 1$ and $\mathrm{P} 2$. Note the sharpness of the cusp at $\mathbf{P} 2$.

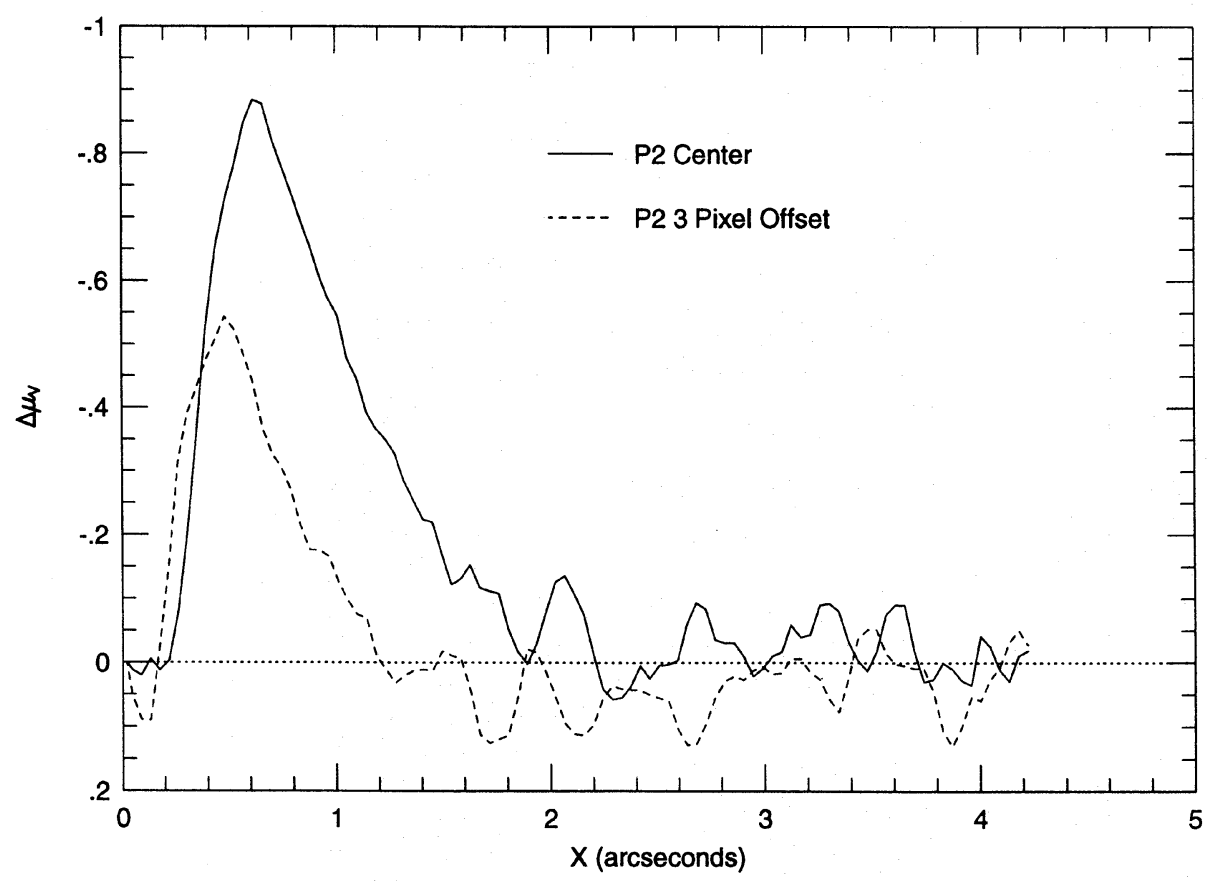

FIG. 7. Tests verifying that $P 2$ is the true center of $M 31$ based on the slice shown in Fig. 6 . The solid trace shows the slice folded about the $\mathrm{P} 2$ center and subtracted from itself. The dashed trace is the same experiment, but with the assumption that the correct center is 3 pixels (0.132) closer to $\mathrm{P} 1$ along the $\mathrm{P} 2-\mathrm{P} 1$ axis. With this center offset, for $r>1.5$, the P2 side clearly oversubtracts the P1 side. 
tracted the opposite sides of the cut from each other reflected about a varying center (see Fig. 7). With P2 as the center there is still a slight bias at distances larger than $2^{\prime \prime}$ from $P 2$ in the sense that the P1 side remains slightly brighter than the $\mathrm{P} 2$ side, but with the center of symmetry taken as a point displaced 3 pixels $(0$ ". 132) towards $\mathrm{P} 1$ from $\mathrm{P} 2$, the residuals at the same location are clearly biased in the sense of over-subtraction. Since the brightness minimum between $\mathrm{P} 2$ and $\mathrm{P} 1$ occurs at 0 ".26 from P2, or twice this distance, the center of symmetry is clearly much closer to the P2 location than the dark zone.

\subsection{Decomposition of the Nucleus}

If $\mathbf{P 2}$ is the true center of the galaxy, then we must explain the prominence of P1. One idea is that P1 is a separate stellar system superimposed on or falling into the nucleus. Alternatively, most of the nucleus may be obscured by dust with $\mathbf{P 1}$ representing a relatively unobscured portion. To explore both possibilities, we attempted to model the nucleus as the simple superposition of two stellar systems, using the multi-isophote decomposition method of Lauer (1986), where one system P1 and the other is P2 plus the bulge (since P2 is coincident with the bulge, we make no distinction between the two for this analysis). The decomposition assumes that each system comprises concentric elliptical isophotes, but with otherwise arbitrary brightness, ellipticity, and position angle profiles; all parameters are solved for simultaneously by a nonlinear least-squares routine.

The decomposition procedure has been "battle-tested" in over 200 multiple-nucleus systems, but it fails to model the M31 nucleus. Figure 8 (Plate 63) shows the results of the decomposition experiments applied to the deconvolved $V$-band image. The basic problem is that the dark zone between P1 and P2 really appears to be a region of "missing light"; no solution was possible that simultaneously fit the isophotes near the dark zone and on the opposite sides of the peaks (the decomposition algorithm became unstable and refused to converge to a solution). If the pixels within the dark zone are ignored by the decomposition algorithm, then a satisfactory solution can be obtained, but one that only ratifies the deficit of light between the two peaks. The main output of the decomposition is a model for the total nucleus, which is shown in Fig. 8; the image divided by the model is also shown in Fig. 8. The dark zone is clearly isolated as a sharply defined region where the image falls below the model, thus showing that the nucleus cannot be explained as the sum of two systems with elliptical and concentric isophotes.

We show the best isolation of the P1 and P2 systems individually from the total nucleus in Fig. 8. As we discuss below, this analysis really depends on a physical picture of what generates the complex structure of the total nucleus, but at present, we can at least show what the individual systems look like if we force both to have elliptical and concentric isophotes and then subtract the individual component fits. We first show an attempt at isolating $P 1$ in the lower left panel of Fig. 8, by subtracting the symmetric P2 model. Ignoring the obvious asymmetry associated with the dark zone (which is assigned completely to P1 if we believe that $\mathbf{P} 2$ really is symmetric), we see that $\mathbf{P} 1$ otherwise appears to be a highly compact, elliptically shaped star cluster. Since the dark zone was excluded from the fit, this actually maximizes the light assigned to P1 and its radial extent, as the $\mathrm{P} 1$ light distribution is then mainly determined from pixels on the side opposite its center from the dark zone. We also show the isolation of P2 in the lower central panel of Fig. 8 under the assumption that symmetrical model for P1 is a correct description of P1.

\subsection{Surface Brightness Profiles}

The decomposition of the nucleus attempted above produces surface brightness profiles of the P2 and P1 components under the assumption of no dust absorption, with the caveat that we have still forced both systems to be symmetric and excluded the dark zone. If in contrast, $\mathrm{P} 2$ is the true, but dust-obscured center, then the observed surface brightness interior to $\sim 1$ ".5, can serve only as a lower limit to the true nuclear surface brightness distribution. We present surface brightness profiles derived from the $V$-band deconvolved image under both assumptions in Figs. 4 and 9 and Table 1.

If the structure of the nucleus is generated by dust absorption, and P2 is the true center, then we get two separate brightness profiles that diverge interior to $\sim 1.5$, depending on which side of the nucleus we measure the profiles from. To measure the profile from the P2 side (which would be partially dust obscured), the pixels dominated by $\mathbf{P} 1$ and the dark zone were excluded. This profile is shown by the solid symbols in Fig. 4. Note that it is actually brighter than the profile derived from the symmetric decomposition. In the decomposition we assumed that P1 overlapped with P2, thus the "no dust" P2 profile (the open symbols in Fig. 4) reflects the underlying P1 contribution subtracted from the total nucleus. Further, since in the dust picture, $\mathbf{P} 2$ is dimmer than $\mathbf{P} 1$ only because it is more obscured, the true profile must be brighter still. If, in fact, $\mathbf{P 1}$ is brighter because it is a portion of the nucleus unaffected by dust, then the $P 1$ side gives a third estimate of at least the outer portion of the nucleus brightness distribution (the dashed line in Fig. 4). For the P1 side, the nucleus rises as a $\gamma \approx-1.8$ power law above the $\mathrm{P} 2$ side profile for $0.7<r<1$."5 (the center is still assumed to be at P2). At $r \sim 0$ ".7, the P1 side flattens off to a constant surface brightness, which would then fall again (not shown) as one moved closer to P2. Under this picture, the flattening of the P1-side brightness profile would be dust induced. One might criticize this whole scenario, however, as the corresponding point on the P2 side still shows a significant brightness gradient, even though the total brightness there is reduced by the greater attenuation presumed to exist on the $\mathrm{P} 2$ side.

The above discussion shows three plausible attempts at estimating the P2 plus bulge profile. In the first picture considered, where $\mathrm{P} 1$ is assumed to be a separate stellar system, the P1 brightness profile generated by the decom- 


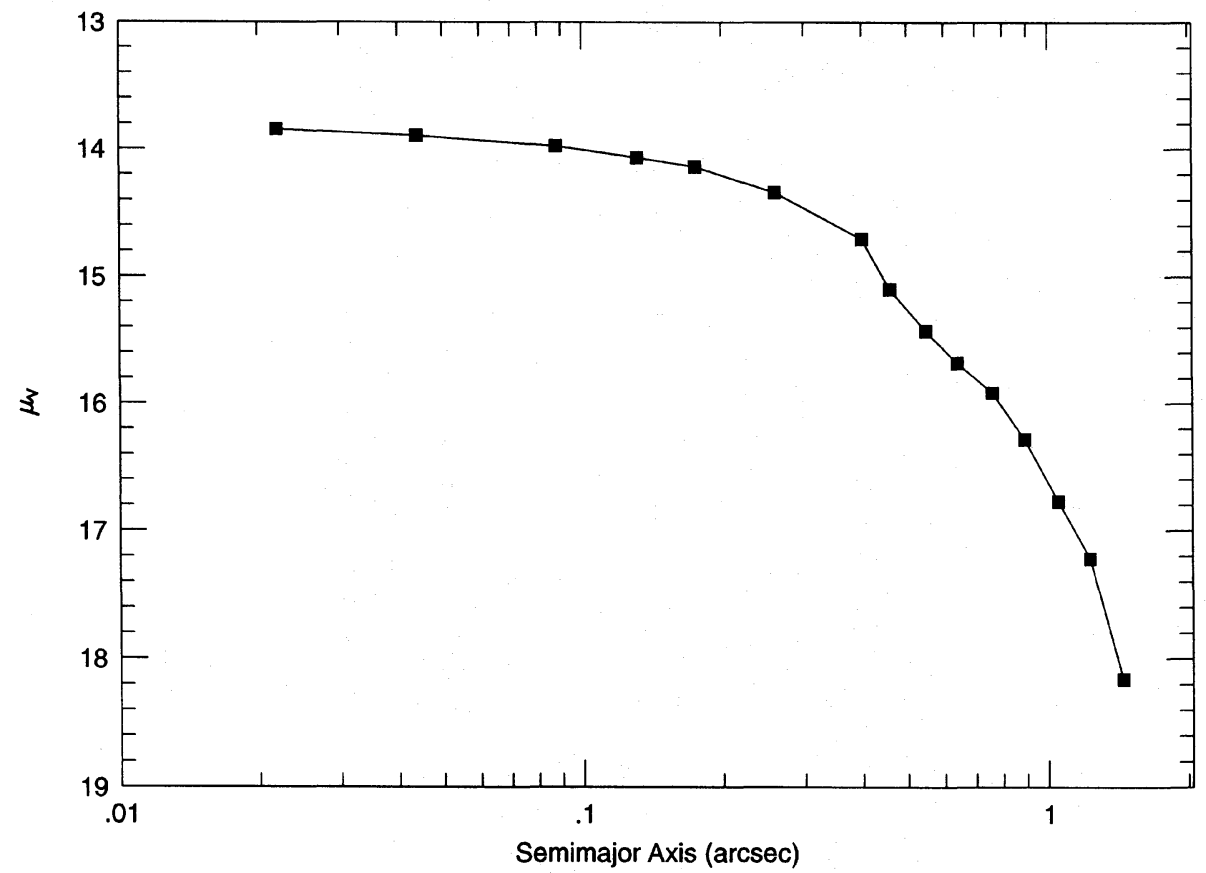

FIG. 9. Deconvolved $V$-band major-axis surface brightness profile for the $P 1$ component. This profile was derived from a simultaneous fit for the P1 and P2 light distributions. The dark zone was excluded from the fit.

TABLE 1. M31 surface brightness profiles.

\begin{tabular}{rcccccccccc}
\hline \hline \multicolumn{1}{c}{ P2 (P1 Clip) } & \multicolumn{3}{c}{ P2 (P1 Fit) } & \multicolumn{3}{c}{ P1 } \\
$\mathrm{R}$ & $\mu_{V}$ & PA & $\epsilon$ & $\mu_{V}$ & PA & $\epsilon$ & $\mu_{V}$ & PA & $\epsilon$ \\
\hline 0.022 & 13.604 & 77.9 & 0.200 & 13.817 & 90.8 & 0.157 & 13.844 & 76.7 & 0.200 \\
0.044 & 13.646 & 77.9 & 0.200 & 13.882 & 90.8 & 0.157 & 13.892 & 76.7 & 0.200 \\
0.088 & 13.714 & 47.3 & 0.200 & 13.957 & 48.8 & 0.200 & 13.975 & 76.7 & 0.200 \\
0.132 & 13.753 & 45.5 & 0.399 & 13.975 & 36.4 & 0.424 & 14.069 & 76.7 & 0.156 \\
0.176 & 13.781 & 44.6 & 0.496 & 13.984 & 34.7 & 0.568 & 14.139 & 60.7 & 0.283 \\
0.260 & 13.807 & 45.5 & 0.633 & 13.974 & 25.8 & 0.627 & 14.339 & 57.5 & 0.301 \\
0.400 & 13.926 & 53.9 & 0.543 & 14.133 & 48.4 & 0.464 & 14.704 & 72.0 & 0.318 \\
0.460 & 14.053 & 61.2 & 0.420 & 14.206 & 59.3 & 0.480 & 15.099 & 71.7 & 0.243 \\
0.550 & 14.142 & 60.3 & 0.429 & 14.302 & 53.0 & 0.469 & 15.429 & 63.3 & 0.231 \\
0.640 & 14.238 & 64.0 & 0.468 & 14.344 & 58.4 & 0.539 & 15.680 & 61.9 & 0.269 \\
0.760 & 14.396 & 62.4 & 0.428 & 14.497 & 57.2 & 0.460 & 15.916 & 63.3 & 0.295 \\
0.890 & 14.561 & 64.8 & 0.405 & 14.647 & 59.0 & 0.430 & 16.281 & 68.2 & 0.323 \\
1.050 & 14.751 & 62.2 & 0.373 & 14.782 & 57.7 & 0.437 & 16.771 & 68.1 & 0.347 \\
1.230 & 14.958 & 59.0 & 0.341 & 14.991 & 55.9 & 0.375 & 17.219 & 64.9 & 0.395 \\
1.450 & 15.124 & 58.5 & 0.317 & 15.185 & 54.4 & 0.301 & 18.161 & 73.0 & 0.327 \\
1.710 & 15.227 & 55.6 & 0.353 & 15.294 & 56.2 & 0.324 & - & - & - \\
2.010 & 15.417 & 55.4 & 0.283 & 15.444 & 56.6 & 0.264 & - & - & - \\
2.360 & 15.548 & 57.3 & 0.264 & 15.546 & 56.7 & 0.261 & - & - & - \\
2.780 & 15.669 & 59.2 & 0.214 & 15.668 & 59.2 & 0.212 & - & - & - \\
3.270 & 15.781 & 49.2 & 0.126 & 15.782 & 51.7 & 0.121 & - & - & - \\
3.850 & 15.879 & 50.6 & 0.134 & 15.878 & 50.2 & 0.138 & - & - & - \\
4.520 & 15.977 & 55.4 & 0.124 & 15.978 & 55.5 & 0.122 & - & - & - \\
5.320 & 16.056 & 55.1 & 0.086 & 16.056 & 54.6 & 0.086 & - & - & - \\
6.260 & 16.125 & 46.7 & 0.126 & 16.125 & 47.3 & 0.124 & - & - & - \\
7.370 & 16.185 & 52.1 & 0.164 & 16.185 & 52.0 & 0.164 & - & - & - \\
8.670 & 16.260 & 43.7 & 0.162 & 16.261 & 43.4 & 0.160 & - & - & - \\
10.200 & 16.325 & 44.0 & 0.213 & 16.323 & 44.6 & 0.216 & - & - & - \\
\hline \hline & & & & & & & & & &
\end{tabular}

Notes to TABLE 1

Major-axis surface brightness profiles are measured from the $V$ band deconvolved image. The first set of columns gives the profile centered on P2 with the excess brightness in P1 and the dark zone clipped out of the images. The second set of columns gives the $P 2$ profile in a simultaneous fit with P1. The last set of columns gives the P1 profile derived from the simultaneous fit. No correction for galactic absorption has been applied. position algorithm gives a description of this component, and is shown in Fig. 9. As already noted above, P1 is sharply limited and its brightness profile falls rapidly, even though the decomposition algorithm is most generous to assigning light to P1 under the "no dust" assumption-if we simply measured the profile of the excess light on the $\mathrm{P} 1$ side compared to the $\mathrm{P} 2$ side, then the resulting profile would be even more truncated. The total $V$ magnitude of the P1 model is $m_{V}=14.5$, which gives $M_{V}=-10.2$ or $L_{V} \approx 1 \times 10^{6} L_{\odot}$ assuming $A_{V}=0^{\mathrm{m}} 24$ (Burstein \& Heiles 1984).

Superficially, both peaks in the M31 nucleus appear to be well resolved. For P1, we get $r_{c}=0 " 37$, or $1.37 \mathrm{pc}$ (this is from the symmetric profile and ignores the dark zone, which is actually interior to the core on one side). For P2 we get $r_{c}=0$ " 82 , or $3.73 \mathrm{pc}$, where we define the core radius simply as the point where the major axis surface brightness falls to half of its central value. The P1 core is computed after subtraction of the underlying bulge; if we measure the core without bulge subtraction, then we agree perfectly with the Light et al. (1974) value $r_{c}=0$ ".48. It must be remembered, however, that Light et al. assumed that P1 was the true nucleus center, thus their profile really should not be compared to those presented here. We also emphasize that use of the term "core" in this context does not necessarily imply that the profile is isothermal. P2, in particular maintains a shallow $\gamma \approx-0.08$, constant power-law brightness gradient for $r<0$ ".2-indeed P2 looks quite cuspy in the linear plot of surface brightness shown in Fig. 6. At the current resolution we cannot say whether there is a shallow stellar cusp or perhaps a weak point source at the very center of the nucleus. The "deconvolved" PSF still 
has finite width, and for $r<0$ ". 1 , there will still be some residual blurring; however, the shallow gradient extends out to twice this distance, showing little evidence that it is really steeper than shown in the innermost portion.

Color information might be useful to unravel the complexity of the M31 nucleus, but given the ambiguities in the surface brightness profiles, extracting colors from this procedure would be highly suspect. Instead we restrict ourselves to a few large-scale measurements that do not depend greatly on the details of the decomposition. We show a color ratio image in Fig. 10 (Plate 64), but unfortunately, this image is more confusing than anything else. The centers of P1 and P2 both appear to be slightly bluer than the average bulge color, but this may be a resolution effect, given that the $I$-band image has poorer resolution. There is no color difference in the dark zone, showing directly the conclusion that the effect of any dust would have to be grey. The one noticeable effect is that the outer portion of $\mathbf{P} 1$ to the east of the major axis appears to be redder than the rest of the image. The structure in this area has not interested us so far-the difference may be due to foreground dust. Overall, the average color for the bulge $\left(r>3^{\prime \prime}\right)$ is $V-I=1.84$, while for P1 $V-I=1.90$, and P2 $V-I=1.73$, where the colors for the peaks refer to apertures 10 pixels in radius with subtraction of the estimated contribution of the other peak (the colors are uncorrected for galactic reddening). The color for the bulge is probably accurate to $0: 01$, while the peak color may differ by as much as $0^{\mathrm{m}} \cdot 1$, depending on how and over what region the magnitudes are measured.

\section{DISCUSSION}

The analysis in the preceding sections presents the basic description of the M31 nucleus against the backdrop of two physical pictures of the nucleus; namely that its structure is due to dust absorption or alternatively to the presence of a separate stellar system. We consider both pictures in turn in this section. At present we cannot decisively choose one picture over the other, thus our tack is to simply lay out what we have considered to sharpen further research.

\subsection{A Dusty Partially Obscured Nucleus}

The outer isophotes of the nucleus are centered on the bulge. The nuclear isophotes become flatter towards the center, beginning some $\approx 3^{\prime \prime}$ out from P2, with the ellipticity at the $P 2$ peak reaching the high value of $\epsilon \sim 0.5$. The nucleus is asymmetric interior to $r \sim 1.5$ to be sure, but even so the nature of the asymmetries do not appear to be random. P1 is also highly elliptical at its center, and its axis is collinear with the line defined by the morphology of P2. The dark zone is sharp and is nearly perpendicular to the major axis. P1 looks sharply cut off in the P2 direction, and in any case cannot be fully modeled as a simple star cluster seen in projection. $\mathbf{P} 1$ is also close to the major axis defined by the outer portions of the nucleus, and its associated excess brightness quickly and smoothly disappears only $\sim 2$ " from P2. The whole picture is roughly consistent with a flattened disk or bar crossed by a perpendicular dust lane.
A problem with this model is that the effects of dust absorption are evidently grey. We find no strong $V-I$ color gradients in the nucleus that we can ascribe to dust, and the $K$-band observations of Mould et al. (1989) make it clear that the $\mathbf{P 1 / P 2}$ asymmetry is just as strong in the near-IR. Mould et al. have already argued that this rules out the nuclear asymmetry as being due to a simple foreground patch of ordinary dust (which is not suggested by the morphology at $H S T$ resolution in any case), but suggest that an optically thick dust torus surrounding the nucleus might still do the trick. This picture would be appealing if $\mathrm{P} 2$ were really just the outer part of the nucleus seen poking out from behind the torus hiding the very center of the nucleus. We believe, however, that P2 in fact is the true center. In the dust picture we would have to explain the $\mathbf{P} 1 / \mathbf{P} 2$ asymmetry by assigning $\mathbf{P} 1$ to the true unaffected outer region of the nuclear light profile, with $\mathrm{P} 2$ heavily attenuated by dust.

We can think of two ways to make color-neutral dust absorption. The first is to have an extremely optically thick screen of ordinary dust, perhaps confined to the plane of the bulge, bisect $\mathrm{P} 2$ but stop short of the P1 side of the nucleus. It is difficult to imagine, however, that the dust patch could terminate without showing strong color effects at its edges. The second possibility is that the dust consists of unusually large grains. One might be able to postulate a more symmetrical arrangement of dust in this case, but the geometry of its distribution would still be complex-some sort of warped plane would be required, which at one point would be seen nearly edge on, forming the dark zone, but would elsewhere cut across the nucleus and screen out most, but not all, of the light on the P2 side. Furthermore, this screen would have to be highly uniform, as the P2 side brightness profile is smooth and maintains a positive gradient all the way into the center, nor is the P2 centroid displaced from the bulge.

While it is unattractive to appeal to unusual dust properties, we must remember that the present $H S T$ observations are the highest resolution optical images ever obtained of a galactic nucleus, a special and still unknown environment. If M31 does have a massive black hole at its center, then it is likely to have had an earlier active phase. Central dust tori are commonly invoked to explain the properties of AGNs and are subjected to strong and unique radiation fields. Laor \& Draine (1993), for example, have argued that the dust in AGNs may indeed have an unusually large $(10 \mu)$ grain size, which would nicely fill our requirement that the dust have no color effect at optical and near-IR wavelengths.

Finally, the relationship between the nuclear kinematics and the dust picture is ambiguous. To first order, the rotation and velocity dispersion traces through the nucleus are symmetric and centered on P2 (Kormendy 1988; Dressler \& Richstone 1988). Complete dust absorption might affect the observed dynamics, but if P2 is only attenuated by thin grey dust rather than truncated by optically thick dust, then the line of sight would still go through most of the nucleus, fairly sampling the stellar field. Kormendy's observations may be consistent with a 
somewhat lower velocity dispersion on the P1 side of the dark zone (a point which we discuss fully in the next section). Since in the dust picture we argue that $\mathrm{P} 1$ is less affected by dust, we would expect any decrease in dispersion to be on the P2 side instead, where dust would be more likely to affect the line-of-sight integral.

\subsection{A Stellar System Orbiting the Nucleus}

If P2 is not attenuated and is the actual nucleus of M31, then P1 must be something extra-an obvious idea is that P1 is a separate stellar system orbiting the nucleus. We further argue that in this picture $\mathbf{P} 1$ must indeed be close to the nucleus. The P1 profile is strongly truncated, and its shape is asymmetric, perhaps suggesting even more severe truncation on its side facing P2. The positioning of P1 on the nucleus major axis, and the relatively symmetric rotation profiles, which shows that P1 sits at the expected velocity location within the nucleus, also suggest that $\mathbf{P} 1$ is really in the nucleus at least, rather than being a chance line-of-sight superposition of a more distant star cluster.

The major problem for the cluster hypothesis is the longevity of any system tightly bound to the nucleus. The orbital time near the P1 location is very short, only $t_{0}=5 \times 10^{4}(r / 2 \mathrm{pc})^{3 / 2}\left(\mathscr{M}_{\odot} / 3 \times 10^{7} \mathscr{M}_{\odot}\right)^{-1 / 2} \mathrm{yr}$, where we have assumed that the central potential is dominated by a black hole. Naive calculations of dynamical friction orbital decay show that P1 would survive for only a few orbital times (Dressler \& Richstone 1988). This would mean that we are now looking at M31 at a very special time. On the other hand, a thorough examination of the dynamics of the $\mathrm{P} 1 / \mathrm{P} 2$ system may show that ordinary dynamical friction is ineffective here. While we cannot identify any mechanism that will allow P1 to survive for a significant fraction of the age of the Universe, we raise the possibility that its life may be substantially longer than its orbital time-if so, then the probability of witnessing the $\mathrm{P} 1 / \mathrm{P} 2$ system in its present state would also be increased substantially.

Dynamical friction works only when there is relative motion between a massive object and a background stellar field. If P1 is bound to the nucleus and is co-orbital with stars in a central disk, then such a drift does not exist. In fact, it is possible that if $\mathrm{P} 1$ is at the edge of a dense stellar disk dominated by a massive black hole at P2, then it may actually draw energy from the disk. If two objects interact gravitationally in a Keplerian velocity field, then the object closer to the center passes energy to the object further out. Additional effects may increase the decay time. Begelman et al. (1980) have considered the orbital decay of a massive black hole into a galactic nucleus already dominated by another pre-existing black hole and conclude that there is a radius at which dynamical friction may shut off. As the black hole binary "hardens," stars within the original nucleus are scattered out of the galaxy core, ultimately depleting the population of stars that could exchange energy with the binary. It is intriguing that the predicted shutoff radius for M31 in this model, $\sim 1 \mathrm{pc}$, closely matches the observed projected separation of $\mathrm{P} 1$ and $\mathrm{P} 2$. All of this makes it clear that the orbital evolution of P1 depends strongly of the detailed underlying nuclear mass distribution and kinematics of the stars and cannot be estimated reliably from any simple dynamical-fraction decay formula.

Even if P1 can maintain a stable orbit, it looks strongly vulnerable to tidal destruction by the central mass in M31. If $\mathbf{P} 1$ is orbiting in a Keplerian potential, then to survive it must have an average mass density $\rho_{1}>(2-f) /$ $(1-f)^{2} \rho_{2}$, where $f$ is the fractional radial extent of $\mathrm{P} 1$ compared to its separation from $\mathrm{P} 2$, and $\rho_{2}$ is the average mass density of $\mathbf{P} 2$ interior to the $\mathrm{P} 1$ orbit. If we assume $f \sim 1 / 3$, a true $\mathrm{P} 1-\mathrm{P} 2$ separation of $3 \mathrm{pc}$ (which assumes a true separation about twice the projected separation), and a black hole mass close to the lower limit of the "nondisk" models, then we require $\rho_{1}>1.0 \times 10^{6}\left(\mathscr{M}_{\odot} / 3 \times 10^{7} \mathscr{M}_{\odot}\right)(3 \mathrm{pc} / r)^{3} \mathscr{M}_{\odot} \mathrm{pc}^{-3}$. This can be compared to the P1 central $V$-band luminosity density of $5 \times 10^{4} L_{\odot} \mathrm{pc}^{-3}$ (the central density for $\mathrm{P} 2$ is $>2 \times 10^{4} L_{\odot} \mathrm{pc}^{-3}$, but is less certain, given its cuspy profile). For the P1 cluster to survive under the present assumptions it must therefore have $\mathscr{M} / L>20$. If the Kormendy (1988) disk picture with $\mathscr{M}_{\bullet \sim 1} \sim 10^{7} \mathscr{M}_{\odot}$ is correct, and we use larger P1-P2 separations, then we can allow for lower $\mathscr{M} / L$ values. On the other hand, we have used the P1 central density-the average density within $2 r_{c}$ is about a factor of 4 lower, which would push us to increase $\mathscr{M} / L$ by a similar factor if we want the outer portions of $\mathbf{P} 1$ to remain bound.

A high-resolution velocity dispersion observation of P1 is crucial to measuring the $\mathscr{M} / L$ of P1. As we alluded to above, it is possible that the Kormendy (1988) and Dressler \& Richstone (1988) velocity dispersion curves may be asymmetric across their origins. If we examine Kormendy's Fig. 4(a) under the assumption that its origin is at $\mathbf{P} 2$, then the dispersion at $\mathbf{P} 1$ is $\sim 180 \mathrm{~km} \mathrm{~s}^{-1}$, while the value at the corresponding point on the opposite side of $\mathbf{P} 2$ is $\sim 230 \mathrm{~km} \mathrm{~s}^{-1}$. We calculate that for the Kormendy (1988) seeing and slit parameters, there are portions of the slit where P1 would contribute $\sim 1 / 3$ of the light to the spectrum. We have simulated spectra of a P1/P2 composite system with differing dispersions in each peak, and conclude that we can always see the effects of P1 if its central dispersion is significantly lower than that of the underlying stars associated with P2. To explain the putative asymmetry in Kormendy's observations, we estimate that the P1 velocity dispersion at Kormendy's ground-based resolution must lie in the range $70-150 \mathrm{~km} \mathrm{~s}^{-1}$. The mass-to-light ratio of a virialized system is $\mathscr{M} / L=9 \sigma^{2}\left(4 \pi G \rho_{L} r_{c}^{2}\right)^{-1}$, where $\rho_{L}$ is the central luminosity density. For $\mathrm{P} 1$, the implied range in $\sigma$ gives $\mathscr{M} / L$ from $10-40$ in solar units. This is consistent with the tidal argument above and, at the upper end, is also comparable to the P2 $\mathscr{M} / L \approx 34$ calculated in the same way.

Both the tidal destruction arguments and kinematics observations lead us to conclude that if $\mathrm{P} 1$ is a separate stellar system, it is not a globular cluster but is rather more likely to be a remnant of a cannibalized galactic nucleus, which are observed to have $\mathscr{M} / L$ ratios within the required range (Lauer 1985; Kormendy 1987). While the 
total P1 $V$-band luminosity is only $L \sim 1 \times 10^{6} L_{\odot}$, which is about as luminous as the most massive globular clusters in M31, globular clusters have only modest mass-to-light ratios and central velocity dispersions. The observations clearly rule out any candidate for $\mathrm{P} 1$ with velocity dispersion as low as that of a globular cluster, a conclusion already reached by Dressler \& Richstone (1988). In fact, it is plausible that $\mathrm{P} 1$ has $\mathscr{M} / L \gg 20$, if we allow higher mass black hole models for $\mathrm{P} 2$, plus the possibility that $\mathrm{P} 1$ has passed as close to $\mathrm{P} 2$ as its projected separation. In this case, we are lead to consider the possibility that P1 contains a central black hole of substantial mass itself. This presents an attractive way to stave off tidal destruction of $\mathbf{P} 1$, and may even be expected if $\mathbf{P} 1$ indeed is a remnant nucleus. On the other hand, if the $\mathrm{P} 1 / \mathrm{P} 2$ system has survived for more than a few orbital times, it may be difficult to push P1 to the highest $\mathscr{M} / L$ ratios allowed by the velocity dispersion observations, since we would not in that case expect the nucleus barycenter to match that of the bulge. From the present location of $\mathrm{P} 2$, we can set a conservative lower limit to the $\mathrm{P} 2 / \mathrm{P} 1$ mass ratio of about $5: 1$, which in turn implies $\mathscr{M} / L \ll 20$, for P1 itself, a result clearly in conflict with the need to have $\mathscr{M} / L$ as high as possible to avoid tidal destruction. One might argue that this is evidence that $\mathbf{P 1}$ is indeed much further from P2 than its projected separation, but in such a case it would be difficult to understand how the $P 1$ radial velocity could still fall on the symmetric M31 nuclear rotation profile.

\section{CONCLUSION}

We have discussed two radical and opposing hypotheses to explain the asymmetric morphology of the M31 nucleus. The first is that the nucleus is a highly flattened bar or disk that is bisected near the center by a thick dust sheet seen nearly edge on. At larger radii the sheet must warp across the line of sight and partially obscure the nucleus. No color effects are seen, so either the dust is extremely optically thick or is comprised of unusually large grains. This is the most unusual component of this explanation, but we cite Laor \& Draine (1993) who were driven to just such a picture to explain the IR spectra of Seyfert nuclei-a plausible connection if M31 was once active. The second hypothesis is that we are seeing the remains of a cannibalized galactic nucleus in the final stages of being absorbed by the M31 nucleus. Longterm survivability of the remnant nucleus in close proximity to a black hole in the nucleus of M31 may require it to have a high $\mathscr{M} / L$ ratio or even a central black hole of its own. This hypothesis requires us to be looking at M31 at a special time; however, the lifetime of P1 against orbital decay may be substantially longer than naive dynamical friction arguments would indicate. Prudence requires us to advance yet another hypothesis, which is that neither hypothesis is correct and that truth has eluded us. To lay groundwork for this contingency, we have attempted to be objective in our basic analysis. We can describe the M31 nucleus at length even if we cannot understand it; any explanation not considered here must be true to this description. We summarize our picture of the M31 nucleus as follows.

- The nucleus is a distinct stellar component at the center of the M31 bulge. The nucleus is evident as an inflection and abrupt rise in the M31 surface brightness profile interior to $3 "$. The isophote ellipticity also increases abruptly interior to this point. The centers of the outer nuclear isophotes are individually coincident with that of the bulge to $\sim 0$ " 1 .

- At the brightest isophotes, or interior to 1.4 , the nucleus becomes asymmetric, having two peaks separated by 0 "49. The brighter of the two peaks, $\mathrm{P} 1$, corresponds to the nuclear core identified by the Stratoscope II pictures obtained by Light et al. (1974). The less luminous peak, P2, is coincident with the photocenter of the bulge to within 0 .05. We argue that this is also the kinematic center of M31 and thus the location of any central black hole. This complex structure shows that the nucleus cannot be explained as a single system displaced from the center of the M31 bulge, or even as a single system with its core sheared out of place with respect to its outer portions.

- Both peaks are highly elongated at their brightest levels. The line defined by both peaks is twisted by $\sim 20^{\circ}$ from the outer nuclear isophotes, but is intermediate in position angle between the M31 bulge and disk. The dark zone between the two peaks is both deeper and sharper than a simple saddle defined by superposition of two elliptically symmetric peaks. Neither peak is elliptically symmetric, and the nucleus cannot be explained as the simple line-ofsight superposition of two stellar systems each with elliptical and concentric isophotes in the absence of any absorbing screen.

- P1 is sharply limited in extent. Its brightness profile is flattened at its center, and it is well resolved, having a core radius of $1.37 \mathrm{pc}$. We argue that if $\mathrm{P} 1$ indeed is a separate stellar system, then it must be in close proximity to the nucleus, rather than being in the distant foreground or background.

- P2 has a shallow brightness gradient that continues into its center.

- The $V$-band image is sharper than the $I$-band image, but otherwise there are no strong differences in $V-I$ between the two peaks and the dark zone that separates them. The asymmetry seen in the ground-based $K$-band images of Mould et al. (1989) appears to be consistent with that discussed here.

An implicit component of our analysis is that the M31 nucleus really does host at least one black hole, of mass $\sim 3-5 \times 10^{7} \mathscr{M} \odot$. Our observations offer no clear proof of this hypothesis, although the morphology of $\mathrm{P} 2$ at least may be suggestive of the rapidly rotating disk advocated by Kormendy (1988). If $\mathbf{P} 1$ is a separate system, however, its continued existence at the center of M31 may require a massive central black hole at $\mathbf{P} 2$. If $\mathbf{P} 2$ were rotating slowly, or had a rotation field decreasing towards it center, then it is hard to imagine any scenario under which P1 could survive for more than a few orbital times. In contrast, if the P1 system could draw energy from the organized velocity field comprising stars closely bound to a 
black hole at $\mathrm{P} 2$, then its lifetime might be greatly enhanced. The viability of our hypothesis that P1 is the remnant of a cannibalized galactic nucleus thus may be closely tied to the issue of whether or not M31 is host to a massive black hole at $\mathrm{P} 2$.

For future work, we hope that a repaired Hubble Space Telescope will obtain observations that can test the hypothesis presented here. The repaired optics should have double the spatial resolution and deliver images with an order of magnitude better dynamic range. If dust is playing a role in shaping the nuclear morphology, suggestive subtle color effects and absorption features that we missed here may become evident. The real test, however, will be to obtain high-resolution velocities and velocity dispersions at $\mathbf{P 1}$, P2, and along the nucleus major axis. First, this would fix the kinematic center of M31 with respect to the morphology of the nucleus presented here-but more importantly, such observations should provide an unequivocal test of the existence of massive black holes at the centers of either $\mathbf{P} 1$ or $\mathbf{P} 2$.

We note in closing the possibility that, over the HST lifetime, proper motions in the M31 nucleus may be detected. The orbital velocity around a black hole at about the projected location of P1 is $350\left(\mathscr{M}_{d} / 5\right.$ $\left.\times 10^{7} \mathscr{M}_{\odot}\right)^{1 / 2}(1.8 \mathrm{pc} / r)^{1 / 2} \mathrm{~km} \mathrm{~s}^{-1}$. If $\mathrm{P} 1$ is orbiting across the line of sight with this velocity, this corresponds to a proper motion of $\sim 10^{-4} \operatorname{arcsec}_{\mathrm{yr}^{-1}}$, a motion that might be detected over the next decade if observations with suitable signal to noise and astrometric references are taken at this epoch.

We thank Dr. Martin Schwarzschild, Dr. Lars Hernquist, Dr. Gerald Quinlan, and Dr. John Kormendy for useful conversations. This research was conducted by the WFPC Investigation Definition Team, supported in part by NASA Grant No. NAS5-1661.

\section{REFERENCES}

Begelman, M. C., Blanford, R. D., \& Rees, M. J. 1980, Nature, 287, 307 Burstein, D., \& Heiles, C. 1984, ApJS, 54, 33

Burrows, C., Holtzman, J. A., Faber, S. M., Bely, P., Husan, H., Lynds, C. R., \& Schroeder, D. 1991, ApJ, 369, L21

de Vaucouleurs, G. 1958, ApJ, 128, 465

Dressler, A. 1984, ApJ, 286, 97

Dressler, A., \& Richstone, D.O. 1988, ApJ, 324, 701

Freedman, W. L., \& Madore, B. F. 1990, ApJ, 365, 186

Gerhard, O. E. 1988, MNRAS, 232, 13P

Griffiths, R. 1989, Wide Field and Planetary Camera Instrument Handbook (ST ScI publication)

Johnson, H. M. 1961, ApJ, 133, 309

Kent, S. M. 1983, ApJ, 266, 562

King, I. R., et al. 1992, ApJ, 397, L35

Kormendy, J. 1987, in Structure and Dynamics of Elliptical Galaxies, IAU Symposium No. 127, edited by T. de Zeeuw (Reidel, Dordrecht), p. 17

Kormendy, J. 1988, ApJ, 325, 128
Kormendy, J., \& Djorgovski, S. 1989, ARA\&A, 27, 235

Laor, A., \& Draine, B. T. 1993, ApJ, 402, 441

Lauer, T. R. 1985, ApJ, 292, 104

Lauer, T. R. 1986, ApJ, 292, 101

Lauer, T. R. 1989a, in Dynamics of Dense Stellar Systems, edited by D. Merritt (Cambridge University Press, Cambridge), p. 97

Lauer, T. R. 1989b, PASP, 101, 445

Lauer, T. R., et al. 1992, AJ, 104, 552

Light, E. S., Danielson, R. E., \& Schwarzschild, M. 1974, ApJ, 194, 257 Lucy, L. B. 1974, AJ, 79, 745

Mould, J., Graham, J., Matthews, K., Soifer, B. T., \& Phinney, E. S. 1989, ApJ, 339, L21

Nieto, J.-L., Macchetto, F. D., Perryman, M. A. C., di Serego Alighieri, S., \& Lelièvre, G. 1986, A\&A, 165, 189

Redman, R. O., \& Shirley, E. G. 1937, MNRAS, 97, 416

Richardson, W. H. 1972, J. Opt. Soc. Am., 62, 52

Richstone, D., Bower, G., \& Dressler, A. 1990, ApJ, 353, 118 


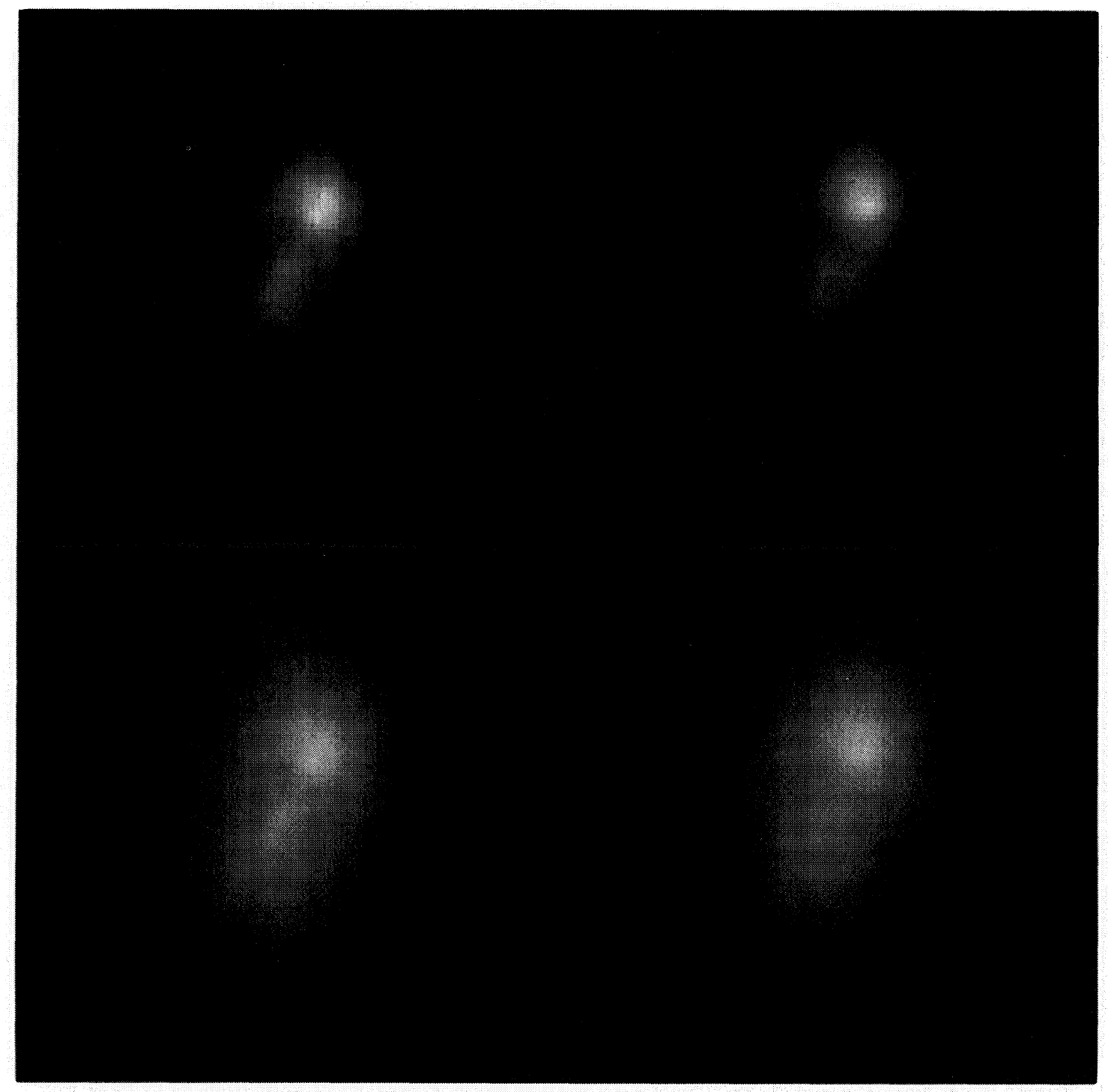

Fig. 1. Planetary Camera images of the M31 nucleus after 80 iterations of Lucy deconvolution. Each panel is 4 " on a side and is centered on P2. The upper two panels are shown with a linear stretch; a logarithmic stretch is used for the bottom two panels. The panels on the left are the $V$-band image (F555W), and the $I$ band (F785LP) is shown on the right. The images have normal celestial handedness. The vertical axis has been rotated $70^{\circ}$ counterclockwise from north.

Lauer et al. (see page 1438)

\section{(c) American Astronomical Society - Provided by the NASA Astrophysics Data System}




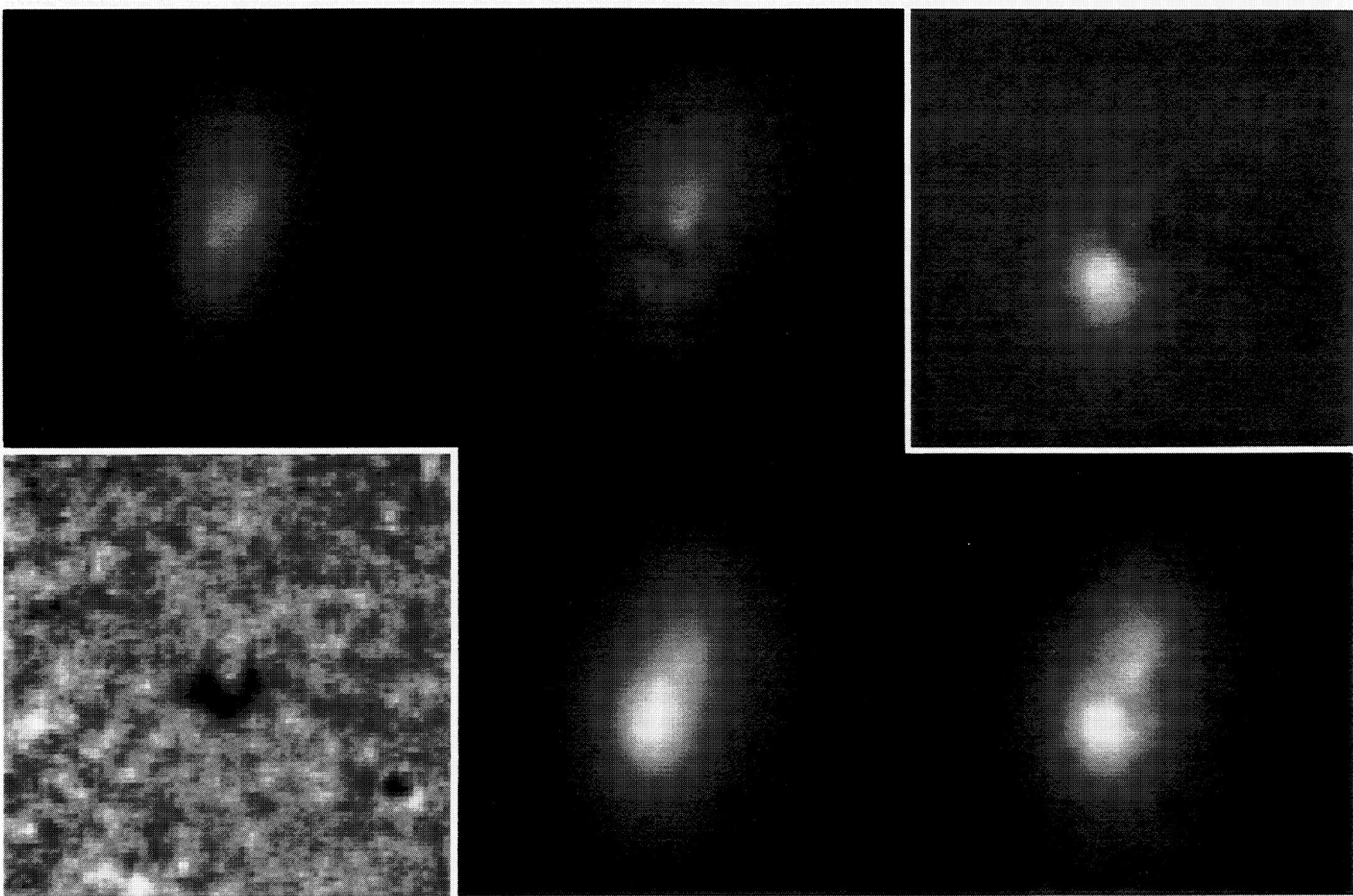

FIG. 8. $V$-band models of the M31 nuclear surface brightness distribution. The location, size, and orientations of the individual panels are as in Fig. 1. The upper left panel is a repeat of the linear representation of the deconvolved $V$-band nucleus image. The upper middle panel is a model of the surface brightness distribution derived by solving for P1 and P2 simultaneously, but excluding the dark zone from the fit. The upper right panel shows the image divided by the model. The stretch is set so that the full range from black to white represents $\pm 25 \%$ deviations of the data with respect to the model. The lower left panel shows an attempt at isolating the P1 component by subtracting the P2 model. A bias has been added to show the background. The next panel shows P2 as estimated by subtracting the P1 model. The last panel shows just the P2 model. 


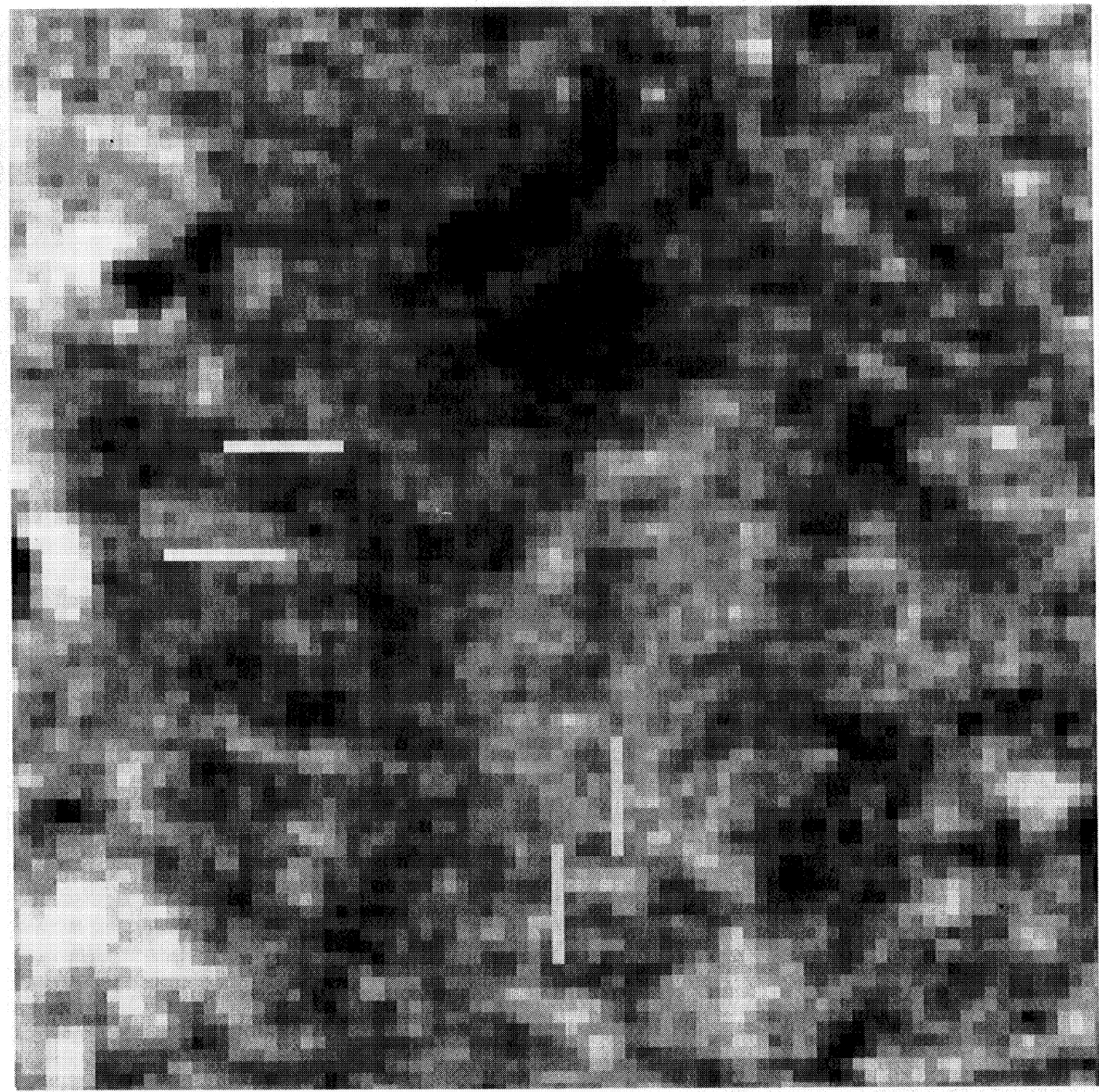

FIG. 10. $V-I$ color map of the M31 nucleus. The image is 4" on a side and is centered on P2. The image has normal celestial handedness. The vertical axis has been rotated $70^{\circ}$ counterclockwise from north (the region shown is identical to that in the individual panels in Fig. 1). The color range from black to white is $0^{\mathrm{m}}$. . Redder colors are darker. Tick marks indicate the peak pixels in $\mathbf{P} 1$ and $\mathbf{P} 2$.

Lauer et al. (see page 1444) 